\title{
AVALIAÇÃO DE FALHAS NO DESCARTE DE RESÍDUOS DOS SERVIÇOS DE SAÚDE
}

\author{
Larissa Teixeira de Oliveira *, Laureane Almeida Santiago de Araujo ** e Ana Claudia Patrocínio * \\ *Universidade Federal de Uberlândia, Uberlândia, Brasil \\ ** Universidade Federal de Uberlândia, Uberlândia, Brasil \\ e-mail: larissateixeiradeoliveira22@gmail.com
}

\begin{abstract}
Resumo: Este trabalho apresenta a geração dos resíduos sólidos de um hospital privado de grande porte na região do Triângulo Mineiro, como é a sua segregação e manuseio, e como os profissionais de saúde portam-se perante o assunto. Durante aproximadamente dois meses os processos de geração de resíduos da instituição foram acompanhados, e, então, observou-se várias falhas na sua segregação. Realizou-se um comparativo com outra Instituição, pública, de grande porte, da mesma região, e analisou-se a grande discrepância nos dados de geração de resíduos infectantes entre eles. Palavras-chave: Resíduos Infectantes, Resíduos Comuns, Conscientização, Segurança do Trabalho
\end{abstract}

Abstract: This paper presents the generation of solid waste in a private hospital in the large region of the "Triângulo Mineiro", as it's segregation and handling, and how health professionals - bear before it. For about two months the waste generation processes of the institution were followed, and then several failures was observed from your segregation. We conducted a comparative with other institution, public, large, the same region, and analyzed the large discrepancy in the data infectious waste generation between them.

Keywords: Infectious waste, common waste, awareness, workplace safety.

\section{Introdução}

Atualmente, mesmo com o avanço da tecnologia, alguns problemas relacionados à Gestão da Segurança, Saúde e Meio Ambiente no Trabalho ainda permanecem, tais como a Infecção Hospitalar e a má Gestão dos Resíduos Sólidos Hospitalares [3, 4].

Ainda hoje no Brasil é comum a incorreta segregação dos resíduos dos serviços de saúde, gerando um tratamento comum para todos eles, o que é inviável, uma vez que a legislação CONAMA 358/05 estabelece que, quando os resíduos infectantes forem adicionados aos comuns, todo o conjunto de resíduos deverá ser tratado como infectante [5]. Tal situação eleva os custos de serviços de saúde, uma vez que tratar todo o resíduo como infectante é inviável para a organização.

A verdadeira situação do Gerenciamento dos Resíduos pode auxiliar no desenvolvimento e no planejamento de medidas preventivas a serem desenvolvidas e trabalhadas na reestruturação do sistema de saúde, além de melhorar a qualidade de vida dos trabalhadores, pautada na higiene, segurança e saúde no trabalho, evitando assim acidentes com os trabalhadores, que podem ocorrer em virtude de um descarte incorreto de pérfuro-cortantes em lixeiras para resíduos comuns e infectantes, além de minimizar os riscos ocupacionais $[3,4,5]$.

$\mathrm{O}$ objetivo deste trabalho é apresentar à instituição de saúde, de grande porte, estudada, os ganhos que ela obterá com ações preventivas em função de ações corretivas, no que diz respeito à Gestão de Resíduos e Biossegurança. A proposta é apontar falhas na segregação e descarte dos resíduos e apresentar os possíveis ganhos, com exemplo de outro hospital de grande porte, com a de correção nos processos, além de atender à legislação.

\section{Materiais e métodos}

A pesquisa descritiva e observacional, de natureza qualitativa foi realizada em uma instituição de saúde privada de grande porte, localizada no interior de Minas Gerais, na região do Triângulo Mineiro, que recebe em média 900 internações por mês, dados contabilizados no ano de 2015. Tem uma média anual de ocupação geral de $72,4 \%$, sendo que na UTI geral teve uma ocupação de $85,9 \%$; na UTI Coronariana, média de 64,9\%; nas Unidades de Internação, média de $76,97 \%$. Neste mesmo ano houve uma média anual de internações por mês de 904 pessoas, e, uma média de atendimentos externos/mês de 4394 pessoas.

Para a produção deste artigo, a coleta de dados foi realizada durante o período de 16/02/16 a 01/04/16, com o objetivo de relacionar e quantificar, além de qualificar, os resíduos gerados no estabelecimento. Neste momento, a pesquisa limitou-se a observar a geração e a segregação de resíduos infectantes nas seguintes unidades: UTI geral, coronária, Pronto Socorro e Centro Cirúrgico, devido à importante quantidade de resíduos gerados nestes setores. Sendo que o estabelecimento de saúde possui um total de 110 leitos, considerando 31 leitos de UTIs, 78 de unidades de internação e 08 leitos no hospital dia.

Em um primeiro momento realizou-se um estudo observacional verificando a sistemática adotada 
pela instituição quanto à segregação e descarte dos resíduos gerados e acondicionamento dos mesmos.

Em um segundo momento, buscou-se por dados quantitativos, a fim de levantar números relacionados à quantidade de resíduos gerados e o custo por quilograma do resíduo de classe A (infectantes) ao hospital.

O intuito principal nesta fase do trabalho é apontar falhas decorrentes da gestão de resíduos do grupo A, uma possível redução de custos diante de uma correta segregação dos Resíduos dos Serviços de Saúde (RSS).

Durante todo o tempo de coleta de informação observou que os RSS, desde o momento em que são gerados, não possuem um manuseio eficaz, uma vez que há inúmeras falhas no processo de segregação, seja por distração do colaborador, falta de sinalização, e, até mesmo, ausência de conhecimento e fiscalização por parte do próprio estabelecimento.

\section{Resultados}

Foram encontradas diversas irregularidades relacionadas à segregação dos RSS, uma vez que a instituição não está atendendo às exigências das legislações brasileiras, resolução 358/05 e 283/01 da CONAMA, RDC 33/03 da ANVISA, NBRs 12 807/93 e $12808 / 93$, o que contribui para a má Gestão de Resíduos Sólidos dos Serviços de Saúde.

As observações quanto ao descarte de resíduos nos setores da UTI Geral, Coronária, Centro Cirúrgico e Pronto Socorro são apresentados na Tabela 1.

Observou-se que há uma deficiência no processo de segregação dos resíduos dos serviços de saúde, gerando o manuseio e descarte incorretos, uma vez que há uma troca no descarte dos resíduos. Além disso, observou-se em leitos de pacientes que não estavam em isolamento apenas lixeiras para resíduos infectantes, ou seja, todo o descarte comum estava sendo feito como Classe A, infectante. E, também, muitas lixeiras nas UTIs encontraram-se lotadas, acima dos limites de tolerância permitidos pela Norma Regulamentadora (NR) 32, do Ministério do Trabalho e Emprego [2].

A Tabela 2 apresenta a quantidade em quilograma dos resíduos do grupo A gerados nos três últimos anos na instituição.

Ao coletar os dados apresentados na Tabela 2, surgiu o questionamento de que possivelmente poderia ter havido alguma mudança na gestão do estabelecimento, ou um aumento no número de atendimentos, porém, realizou-se uma entrevista com os colaboradores, e observou-se que houve, sim, um aumento no número de atendimentos, além de ter havido uma ampliação na estrutura do prédio no final de 2014, justificando o aumento na geração de resíduos.
Tabela 1: Descartes Incorretos dos Resíduos na Instituição.

\begin{tabular}{|c|c|c|}
\hline \multicolumn{3}{|c|}{ Descarte Incorreto de Resíduos } \\
\hline Setor & Resíduo Infectante & $\begin{array}{l}\text { Resíduo } \\
\text { Comum }\end{array}$ \\
\hline UTI Geral & $\begin{array}{l}\text { Papel; plástico; } \\
\text { fraldas de pacientes } \\
\text { não isolados; luvas de } \\
\text { procedimento } \\
\text { simples; capotes, } \\
\text { máscaras, toucas } \\
\text { utilizados em leitos } \\
\text { de pacientes não } \\
\text { is o la d o s; co p o } \\
\text { plástico; papel que } \\
\text { embala sabonete; } \\
\text { sabonete em barra; } \\
\text { fralda de paciente não } \\
\text { isolado (com água } \\
\text { proven i en te do } \\
\text { banho); embalagem } \\
\text { de soro; papel toalha; } \\
\text { algodão; restos } \\
\text { alimentares; }\end{array}$ & $\begin{array}{l}\text { Capotes } \\
\text { utilizados em } \\
\text { le itos d e } \\
\text { pacientes } \\
\text { isolados; gaze } \\
\text { com sangue; }\end{array}$ \\
\hline $\begin{array}{c}\text { UTI } \\
\text { Coronariana }\end{array}$ & $\begin{array}{l}\text { Guardanapo; garfo; } \\
\text { restos de alimentos; } \\
\text { garrafa d'água; }\end{array}$ & Agulha; \\
\hline $\begin{array}{l}\text { Centro } \\
\text { Cirúrgico }\end{array}$ & $\begin{array}{l}\text { Papel; plástico; luvas } \\
\text { de proc edimento } \\
\text { s i m p l e s; c o p o } \\
\text { plástico; embalagem } \\
\text { de bisturi (plástico); }\end{array}$ & $\begin{array}{l}\text { Algodão com } \\
\text { sangue; gaze } \\
\text { com sangue; } \\
\text { luva s d e } \\
\text { procedimento } \\
\text { com sangue; }\end{array}$ \\
\hline $\begin{array}{l}\text { Pronto } \\
\text { Socorro }\end{array}$ & $\begin{array}{l}\text { L u v a s } \\
\text { procedimento } \\
\text { simples; papel; } \\
\text { plástico; rolo de fita } \\
\text { a desiva; papel } \\
\text { higiênico; }\end{array}$ & $\begin{array}{l}\text { Algodão com } \\
\text { sangue; }\end{array}$ \\
\hline
\end{tabular}

Tabela 2: Dados (resíduos infectantes) coletados dos anos de 2013, 2014, 2015 da instituição em estudo.

\begin{tabular}{|c|c|c|c|}
\hline ANO & 2013 & 2014 & 2015 \\
\hline TOTAL & 40625,07 & 47641,37 & 61958,71 \\
EM KG & & & \\
\hline
\end{tabular}

De acordo com a Tabela 2, observa-se que de 2013 para 2014 houve um aumento de aproximadamente $18 \%$ na geração de resíduos infectantes. De 2014 para 2015 houve um aumento mais significativo ainda, de $30 \%$, ou seja, estes dados provam que as medidas de controle (treinamentos, trabalho de conscientização e cursos de capacitação) desenvolvidas 
com os colaboradores não estão sendo eficazes, pois não estão atingindo os reais geradores dos resíduos de Classe A.

Em seguida, foram coletados os dados deste ano, 2016, para que pudesse ser realizada uma estimativa do possível resultado para o final do ano. A Tabela 3 apresenta os dados coletados nos dois primeiros meses de 2016.

Nos dois meses os valores encontrados já representam cerca de aproximadamente $23 \%$ do total produzido no ano anterior, o que reforça que possivelmente, haverá um novo aumento nos dados.

Tabela 3: Dados coletados nos dois primeiros meses do ano de 2016 no estabelecimento em estudo.

\begin{tabular}{|c|c|}
\hline Mês (2016) & Total em KG \\
\hline Janeiro & 9363,11 \\
\hline Fevereiro & 4784,28 \\
\hline
\end{tabular}

Percebe-se que houve uma diminuição da geração de resíduos Classe A de Janeiro para Fevereiro deste ano, de aproximadamente $50 \%$, o que se deu devido a redução do número de atendimentos no estabelecimento.

Para efeitos comparativos a Tabela 4 mostra dados de um Hospital Público de grande porte, localizado na mesma região da instituição em estudo, e apresenta os valores de referência dos resíduos infectantes gerados no ano de 2010 e 2013, no qual no período foram implantadas medidas de conscientização e ações pontuais para a gestão adequada de resíduos com uma proposta de melhorias durante o período dos três anos.

O estabelecimento de grande porte, que atende vários municípios, além de ser hospital escola, utilizado como comparativo para este estudo, propôs treinamentos aos colaboradores, incluindo médicos, enfermeiros, pessoal da limpeza, recursos humanos, diretoria, etc, a fim de apresentar os dados coletados com os resíduos infectantes no ano de 2010, e durantes os anos de 2011, 2012 e 2013 trabalharam com as medidas de controle solicitadas pela NR 32 treinamentos e capacitações - apresentando os tipos de resíduos, como devem ser tratados desde a segregação até a sua disposição final, e obtiveram como resultado uma queda de aproximadamente $29 \%$ na produção dos resíduos de Classe A. Este foi um trabalho realizado pelo SESMT (Serviços Especializados de Segurança e Medicina do Trabalho) do estabelecimento, juntamente com a equipe de Meio Ambiente, que nesta instituição eram equipes separadas, mas que normalmente, são unicamente compostas pelo SESMT, conhecida como Saúde, Segurança e Meio Ambiente no Trabalho, SSMA.

Esta instituição pública trabalha com a política de que falhas causadas pelos seus colaboradores, incluindo os profissionais de saúde causam impactos ambientais e perdas financeiras para a instituição.

A instituição conta com 520 leitos totais, sendo uma referência para 86 municípios da macro e micro região do Triângulo Norte. Atende em média 6000 pacientes por dia.

Tabela 4 Dados coletados a título de comparação, em um hospital público de grande porte da região.

\begin{tabular}{|c|c|c|}
\hline ANO & 2010 & 2013 \\
\hline TOTAL EM KG & 10850 & 7660 \\
\hline
\end{tabular}

\section{Discussão}

Devido aos microrganismos transportados nos Resíduos Infectantes dos Serviços de Saúde (RSS), serem potencialmente infectantes, ou capazes de proliferarem no meio ambiente, hoje, discute-se bastante a respeito da relação entre o Gerenciamento de Resíduos dos Serviços de Saúde e a Infecção Hospitalar, juntamente com os Acidentes de Trabalho (AT) nos serviços de saúde [4].

De acordo com a CONAMA 358/05 os estabelecimentos de serviços de saúde devem ser estimulados a minimizar a geração de resíduos, desde a sua geração até a destinação final. Uma vez que há a segregação dos resíduos, no momento e local de sua geração, haverá uma redução no volume de resíduos que necessitam de tratamento diferenciado, ou seja, resíduos infectantes (Classe A), além de reduzir drasticamente os custos com estes resíduos para o empregador.

Se a produção de resíduos infectantes continuar na mesma proporção durante todo o ano de 2016 (Tabela 3) na instituição privada em estudo neste trabalho, uma estimativa para o total produzido ao final deste ano será de $84884,34 \mathrm{~kg}$, considerando que nestes dois primeiros meses o total foi de $14147,39 \mathrm{Kg}$; ao fazer a média destes meses e, multiplicar por 12, obteremos um possível resultado para o total do ano. Portanto, deve-se observar que este valor é maior do que nos últimos três anos analisados, provando que desde 2015 há uma produção elevada de resíduos Classe A, necessitando de alguma medida corretiva de emergência, e posteriormente treinamentos a serem realizados com todos os colaboradores, inclusive os cotistas da instituição, a fim de minimizar os valores encontrados, considerando que são os que têm as principais participações nos lucros da instituição, e, também, contribuem para a produção dos resíduos e diretamente na sua má segregação, uma vez que são, em sua maioria, profissionais da saúde que fazem parte do corpo de colaboradores da instituição.

A gestão dos RSS deveria ser acompanhada diariamente, em diversos horários durante a jornada de trabalho, a fim de evitar os transtornos encontrados, e, também, promover treinamentos e capacitações aos colaboradores envolvidos nos processos e gerações dos resíduos, uma vez que no estabelecimento é comum a 
segregação incorreta, devido à falta de treinamento e de PGRSS eficientes.

Percebe-se nitidamente a discrepância nos números dos resíduos gerados no estabelecimento em estudo, e naquele comparado (Hospital Público). Neste, estão presentes todos os setores relacionados à Segurança do Trabalho, incluindo SESMT completo, Gestão Ambiental, e observa-se a eficácia dos mesmos, uma vez que não só toda a documentação está em dia, como também trabalhos de conscientização e treinamentos, envolvendo todos os profissionais da instituição.

\section{Conclusão}

O gasto que a instituição tem com o descarte de resíduos está diretamente relacionado aos resíduos infectantes, que têm tratamento específico, mesmo que haja um descarte inadequado.

Portanto, precisa-se introduzir uma metodologia de ensino e trabalho a todos os profissionais do estabelecimento, indiscriminadamente, uma vez que todos eles são responsáveis pela geração dos RSS nos estabelecimentos de saúde.

Além de tudo isso, é importante realizar uma coleta seletiva nos setores analisados, pois irá ajudar a controlar a segregação dos resíduos comuns, tentando evitar o seu descarte no local de resíduos infectantes.

\section{Agradecimentos}

Agradecemos à instituição em estudo, pela oportunidade de realizar a pesquisa.

\section{Referências}

[1] Brasil, Ministério da Saúde. Agência Nacional de Vigilância Sanitária. Manual de gerenciamento de resíduos de serviços de saúde. Brasília: Ministério da Saúde, 2006.

[2] Brasil. Norma Regulamentadora $\mathrm{n}^{\circ} 32$, de 16 de novembro de 2005. Segurança e Saúde no Trabalho em Serviços de Saúde. Brasília, DF, 16 nov. 2005.

[3] Costa, Wesley Moreira da; Fonseca, Maria Christina Grimaldi da. A importância do gerenciamento dos resíduos hospitalares e seus aspectos positivos para o meio ambiente. Revista Brasileira de Geografia Médica e da Saúde, Uberlândia, v. 5, n. 9, p.12-31, dez. 2009.

[4] Garcia, Leila Posenato; Zanetti-Ramos, Betina Giehl. Gerenciamento dos resíduos de serviços de saúde: uma questão de biossegurança. Cad. Saúde Pública, Florianópolis, v. 3, n. 20, p.744-752, maio 2004.

[5] Valério, Mirian Charello; Castanheira, Nelson Pereira. Análise quali-quantitativa do lixo produzido em hospital público do paraná: viabilidade econômica através da correta segregação de materiais recicláveis. meio Ambiente e Sustentabilidade, Curitiba, v. 4, n. 2, p.44-65, jul. 2013.
[6] Conselho Nacional do Meio Ambiente. Resolução n. ${ }^{\circ}$ 006, 19 de setembro de 1991. Diário oficial da República Federativa do Brasil, Brasília, DF,1991.

[7] Conselho Nacional do Meio Ambiente. Resolução n. ${ }^{\circ}$ 005, 5 de agosto de 1993. Define os procedimentos mínimos para o gerenciamento de resíduos sólidos provenientes de serviços de saúde, portos e aeroportos. Diário oficial da República Federativa do Brasil, Brasília, DF,1993.

[8] . Conselho Nacional do Meio Ambiente. Resolução n. ${ }^{\circ}$ 283, 12 de julho de 2001. Dispõe sobre o tratamento e a destinação final dos resíduos dos serviços de saúde. Diário oficial da República Federativa do Brasil, Brasília, DF, 2001.

[9]__. Agência Nacional de Vigilância Sanitária. Resolução RDC N. ${ }^{\circ}$ 33, 25 de fevereiro de 2003. Dispõe sobre o Regulamento Técnico para o gerenciamento de serviços de saúde. Diário oficial da República Federativa do Brasil, Brasília, DF, 05 mar. 2003.

[10] . Agência Nacional de Vigilância Sanitária. Resolução RDC N. ${ }^{\circ}$ 306, de 07 de dezembro de 2004. Dispõe sobre o Regulamento Técnico para o gerenciamento de resíduos de serviços de saúde. Diário oficial da República Federativa do Brasil, Brasília ,DF, 2003.

[11] Conselho Nacional do Meio Ambiente. Resolução n. ${ }^{\circ}$ 358, 29 de abril de 2005. Dispõe sobre o tratamento e a disposição final dos resíduos dos serviços de saúde e dá outras providências. Diário oficial da República Federativa do Brasil, Brasília, DF, 04 maio de 2005. 